\title{
Killing-Yano Tensors, Rank-2 Killing Tensors, and Conserved Quantities in Higher Dimensions
}

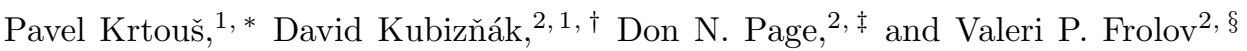 \\ ${ }^{1}$ Institute of Theoretical Physics, Charles University, V Holešovičkách 2, Prague, Czech Republic \\ ${ }^{2}$ Theoretical Physics Institute, University of Alberta, Edmonton, Alberta, Canada T6G 2G7
}

(Dated: December 4, 2006)

\begin{abstract}
From the metric and one Killing-Yano tensor of rank $D-2$ in any $D$-dimensional spacetime with such a principal Killing-Yano tensor, we show how to generate $k=[(D+1) / 2]$ Killing-Yano tensors, of rank $D-2 j$ for all $0 \leq j \leq k-1$, and $k$ rank-2 Killing tensors, giving $k$ constants of geodesic motion that are in involution. For the example of the Kerr-NUT-AdS spacetime (hep-th/0604125) with its principal Killing-Yano tensor (gr-qc/0610144), these constants and the constants from the $k$ Killing vectors give $D$ independent constants in involution, making the geodesic motion completely integrable (hep-th/0611083). The constants of motion are also related to the constants recently obtained in the separation of the Hamilton-Jacobi and Klein-Gordon equations (hep-th/0611245).
\end{abstract}

PACS numbers: 04.70.Bw, 04.50.+h, 04.20.Jb

Alberta-Thy-16-06

\section{INTRODUCTION}

In four-dimensional spacetimes like the Kerr metric [1], the existence of conserved quantities for geodesics (constants of motion) [2] and the tensorial structures that generate them (Killing vectors, Killing tensors [3, 4], and Killing-Yano tensors [5, 6, 7]) have been very important, not only elucidating particle motion in these spacetimes, but also leading to the separation of the Klein-Gordon [2], massless neutrino [8, 9], massive Dirac 10, 11], electromagnetic [12], and gravitational wave [12] equations.

With the recent interest in higher-dimensional spacetimes, it has become of interest to extend these old fourdimensional studies to higher dimensions $D$. For example, it has been found that the rotating black hole metrics [13, 14, 15, 16, 17, 18] in higher dimensions have a Killing-Yano tensor of rank $D-2$ [19, 20] (which we shall call a principal Killing-Yano tensor) that was used (along with the Killing vectors) to show [21, 22] that geodesic motion in the general $D$-dimensional Kerr-NUT-AdS rotating black hole spacetime [18] is completely integrable, with $D$ independent constants of motion in involution.

For convenience, we use square brackets to denote the integer part of what is inside and define $n \equiv[D / 2]$, $k \equiv[(D+1) / 2]$, and $\varepsilon \equiv k-n(0$ for even $D$ and 1 for odd $D)$, so $D=2 n+\varepsilon=2 k-\varepsilon=k+n$. Then the Kerr-NUT-AdS spacetimes have $k$ Killing vectors (giving constants of motion linear in the velocity) and $n$ independent Killing tensors of higher rank (including the metric) [21, 22] (giving other independent constants of motion in involution that are higher-order polynomials in the velocity).

Here we show how to construct $k$ Killing-Yano tensors,

\footnotetext{
*Electronic address: Pavel.Krtous@mff.cuni.cz

${ }^{\dagger}$ Electronic address: kubiznak@phys.ualberta.ca

${ }^{\ddagger}$ Electronic address: don@phys.ualberta.ca

$\S$ Electronic address: frolov@phys.ualberta.ca
}

of ranks $D-2 j$ for $0 \leq j \leq k-1$, for any spacetime with a principal Killing-Yano tensor. Contractions of each of these with itself (leaving two indices free) give $k$ rank-2 Killing tensors and hence $k$ independent constants of geodesic motion in involution for any spacetime with a principal Killing-Yano tensor.

For the case of the Kerr-NUT-AdS spacetimes [18], all of the $k$ Killing vectors can also be constructed from the principal Killing-Yano tensor and its covariant derivative, so all $D$ constants of motion arise from one single Killing-Yano tensor (and the metric, of course, for defining covariant derivatives and contractions). For these metrics, we exhibit explicitly the resulting $k$ Killing-Yano tensors of rank $D-2 j$, the $k$ rank-2 Killing tensors, and the $k$ Killing vectors. (For odd $D, \varepsilon=1$, one of these rank-2 Killing tensors is the tensor product of a Killing vector with itself and so is not independent or irreducible, leaving only $D=2 k-\varepsilon$ independent rank-2 and rank-1 Killing tensors) We also show the relations of the constants of motion arising from all these Killing tensors with those given in [21, 22], as well as with the constants of motion arising from the recent separation of the Hamilton-Jacobi and Klein-Gordon equations [23].

\section{GENERATING FUNCTION}

The following construction of the Killing tensors, Killing-Yano tensors, and of the corresponding conserved quantities applies for any metric [24] with a rank-2 closed conformal Killing-Yano tensor $\boldsymbol{h}$ (or, equivalently, a $(D-2)$-rank Killing-Yano tensor $\boldsymbol{f}=* \boldsymbol{h}$; see Section [V]. Since $\boldsymbol{h}$ and $\boldsymbol{f}$ play an important role in our construction, we call both of them principal tensors.

Let us recall that a rank-2 conformal Killing-Yano tensor $\boldsymbol{h}$ is an antisymmetric 2 -form which obeys

$$
\nabla_{(a} h_{b) c}=\frac{1}{D-1}\left(g_{a b} \nabla_{e} h_{c}^{e}-\nabla_{e} h_{(a}^{e} g_{b) c}\right) .
$$

Assuming the existence of such a tensor, we define the 
2-form $\boldsymbol{F}=\boldsymbol{u} \cdot *(\boldsymbol{u} \cdot * \boldsymbol{h})$, which in components reads

$$
F_{a b}=w h_{a b}-u_{a} u^{c} h_{c b}-h_{a c} u^{c} u_{b}=w h_{c d} P_{a}^{c} P_{b}^{d} .
$$

Here $u^{a}$ is the velocity [25], $w=u_{a} u^{a}$, and $P^{c}{ }_{a}=\delta^{c}{ }_{a}-w^{-1} u^{c} u_{a}$ is the projector to the space orthogonal to $\boldsymbol{u}$. The 2 -form $\boldsymbol{F}$ is covariantly conserved in the direction of $\boldsymbol{u}$,

$$
u^{a} \nabla_{a} F_{b c}=0 .
$$

Now we introduce the generating function $W(\beta)$,

$$
W(\beta)=\operatorname{det}\left(I+\sqrt{\beta} w^{-1} F\right),
$$

where we take $F$ (and similarly $h, P, p$, and $Q$ below) to be the matrix of components $F^{a}{ }_{b}$ of the 2 -form $\boldsymbol{F}$. Due to the antisymmetry of $F$ and properties of the determinant, $W(\beta)$ can be rewritten as a function of $\beta$ instead of $\sqrt{\beta}$, and in terms of $h$ and $P$ instead of $F$,

$$
W(\beta)=\operatorname{det}^{1 / 2}\left(I-\beta w^{-2} F^{2}\right)=\operatorname{det}(I-\sqrt{\beta} h P) .
$$

Because it is constructed only in terms of covariantly conserved quantities $\boldsymbol{F}$ and $w$, the generating function is conserved along geodesics, and the same is true for its derivatives with respect to $\beta$. We can thus define constants of motion $c_{j}$ as the coefficients in the $\beta$-expansion of $W(\beta)$ :

$$
W(\beta)=\frac{1}{w} \sum_{j=0}^{\infty} c_{j} \beta^{j} .
$$

It turns out that all terms with $j>n$ are zero.

To evaluate the observables $c_{j}$, we first split $W(\beta)$ in the following way:

$$
W(\beta)=W_{0}(\beta) \Sigma(\beta),
$$

with

$$
\begin{aligned}
& W_{0}(\beta)=\operatorname{det}(I-\sqrt{\beta} h), \\
& \Sigma(\beta)=\operatorname{det}\left(I+\frac{\sqrt{\beta} h}{I-\sqrt{\beta} h} p\right)=\operatorname{tr}\left((I-\sqrt{\beta} h)^{-1} p\right) .
\end{aligned}
$$

Here $p^{a}{ }_{b}=w^{-1} u^{a} u_{b}$ is the projector into the $\boldsymbol{u}$ direction, and we used the fact that the matrix in the determinant in the expression for $\Sigma(\beta)$ differs from $I$ only in the onedimensional subspace given by $\boldsymbol{u}$. The generating function thus splits into a part $W_{0}(\beta)$ independent of $\boldsymbol{u}$ and a part $\Sigma(\beta)$ linear in $p$. Using the antisymmetry of $h$, we can rewrite $W_{0}(\beta)$ and $\Sigma(\beta)$ in terms of the conformal Killing tensor with components $Q^{a}{ }_{b}=-h^{a}{ }_{c} h^{c}{ }_{b}$,

$$
\begin{aligned}
W_{0}(\beta) & =\operatorname{det}^{1 / 2}(I+\beta Q), \\
\Sigma(\beta) & =\operatorname{tr}\left((I+\beta Q)^{-1} p\right)=\sum_{j=0}^{\infty}(-1)^{j} \operatorname{tr}\left(Q^{j} p\right) \beta^{j} .
\end{aligned}
$$

We shall assume that $h$ is non-degenerate with different eigenvalues. This means that there exist $n$ uniquely defined 2-dimensional subspaces labeled by the index $\mu=1, \ldots, n$, each of which can be spanned by a pair of the orthonormal vectors $\boldsymbol{e}_{\mu}$ and $\boldsymbol{e}_{\hat{\mu}}(\hat{\mu} \equiv n+\mu)$, in odd number of dimensions complemented with a one-dimensional subspace spanned by the vector $\boldsymbol{e}_{\hat{0}}$ $(\hat{0} \equiv 2 n+1)$, with non-zero different eigenvalues $x_{\mu}$, such that $\boldsymbol{h}$ has the form

$$
\boldsymbol{h}=\sum_{\mu=1}^{n} x_{\mu} \boldsymbol{\omega}^{\mu}
$$

Here, $\boldsymbol{\omega}^{\mu} \equiv \boldsymbol{e}^{\mu} \wedge \boldsymbol{e}^{\hat{\mu}}$ are mutually orthogonal 2-forms associated with the 2-dimensional planes.

Using this frame we can write also the conformal Killing tensor $\boldsymbol{Q}$ as

$$
\boldsymbol{Q}=\sum_{\mu=1}^{n} x_{\mu}^{2}\left(\boldsymbol{e}^{\mu} \boldsymbol{e}^{\mu}+\boldsymbol{e}^{\hat{\mu}} \boldsymbol{e}^{\hat{\mu}}\right)
$$

Now we can write down the functions $W_{0}$ and $\Sigma$ in terms of the eigenvalues $x_{\mu}$. For the part independent of $\boldsymbol{u}$, we get

$$
W_{0}(\beta)=\prod_{\mu=1}^{n}\left(1+\beta x_{\mu}^{2}\right)=\sum_{j=0}^{n} A^{(j)} \beta^{j}
$$

where (cf. [18], though here in a more general situation)

$$
A^{(j)} \equiv \sum_{\nu_{1}<\cdots<\nu_{j}} x_{\nu_{1}}^{2} \ldots x_{\nu_{j}}^{2} .
$$

Similarly,

$$
\begin{aligned}
\Sigma(\beta) & =\frac{1}{w}\left(\varepsilon u_{\hat{0}}^{2}+\sum_{\mu=1}^{n} \frac{u_{\mu}^{2}+u_{\hat{\mu}}^{2}}{1+\beta x_{\mu}^{2}}\right) \\
& =\frac{1}{w}\left(\varepsilon u_{\hat{0}}^{2}+\sum_{j=0}^{\infty}(-1)^{j} \beta^{j} \sum_{\mu=1}^{n}\left(u_{\mu}^{2}+u_{\hat{\mu}}^{2}\right) x_{\mu}^{2 j}\right),
\end{aligned}
$$

with $u_{\mu}, u_{\hat{\mu}}$, and $u_{\hat{0}}$ being components of $\boldsymbol{u}$ with respect to the dual frame $e^{\mu}, \boldsymbol{e}^{\hat{\mu}}, \boldsymbol{e}^{\hat{0}}$. Recall that $\varepsilon=0$ in even dimensions $D=2 n$ and $\varepsilon=1$ in odd dimensions $D=$ $2 n+1$.

The original generating function reads

$$
W(\beta)=\frac{1}{w} \sum_{j=0}^{n}\left(\sum_{l=0}^{j}(-1)^{l} A^{(j-l)} w_{l}\right) \beta^{j},
$$

where

$$
w_{l}=w \operatorname{tr}\left(Q^{l} p\right)=\boldsymbol{u} \cdot \boldsymbol{Q}^{l} \cdot \boldsymbol{u}=u_{a_{1}} Q^{a_{1}}{ }_{a_{2}} \ldots Q^{a_{l}}{ }_{a} u^{a}
$$

are quantities quadratic in the velocity $\boldsymbol{u}$ given by the $l$-th matrix power $\boldsymbol{Q}^{\cdot l}$ of the conformal Killing tensor $\boldsymbol{Q}$. Clearly, $w_{0}=w$, and $w_{j}=\sum_{\mu}\left(u_{\mu}^{2}+u_{\hat{\mu}}^{2}\right) x_{\mu}^{2 j}$ for $j>0$. In 
terms of the eigenvalues $x_{\mu}$, from the product of Eqs. (12) and (14) we obtain

$$
W(\beta)=\frac{1}{w} \sum_{j=0}^{n}\left(\varepsilon A^{(j)} u_{\hat{0}}^{2}+\sum_{\mu=1}^{n} A_{\mu}^{(j)}\left(u_{\mu}^{2}+u_{\hat{\mu}}^{2}\right)\right) \beta^{j},
$$

where we have introduced the quantities (cf. [18])

$$
A_{\mu}^{(j)} \equiv \sum_{\substack{\nu_{1}<\cdots<\nu_{j} \\ \nu_{i} \neq \mu}} x_{\nu_{1}}^{2} \ldots x_{\nu_{j}}^{2}
$$

\section{CONSTANTS OF MOTION AND RANK-2 KILLING TENSORS}

Comparing Eqs. (15) and (17) with Eq. (6), we can identify the $k=n+\varepsilon=[(D+1) / 2]$ conserved quantities $c_{j}$ (constants of geodesic motion, $j=0, \ldots, k-1$ ),

$$
c_{j}=\sum_{l=0}^{j}(-1)^{l} A^{(j-l)} w_{l}=\varepsilon A^{(j)} u_{\hat{0}}^{2}+\sum_{\mu=1}^{n} A_{\mu}^{(j)}\left(u_{\mu}^{2}+u_{\hat{\mu}}^{2}\right) .
$$

These constants are quadratic in the velocities. They can be generated [4] by rank-2 Killing tensors $\boldsymbol{K}^{(j)}$ as

$$
c_{j}=K_{a b}^{(j)} u^{a} u^{b}
$$

where

$$
\begin{aligned}
\boldsymbol{K}^{(j)} & \equiv \sum_{l=0}^{j}(-1)^{l} A^{(j-l)} \boldsymbol{Q}^{\cdot l} \\
& =\varepsilon A^{(j)} \boldsymbol{e}^{\hat{0}} \boldsymbol{e}^{\hat{0}}+\sum_{\mu=1}^{n} A_{\mu}^{(j)}\left(\boldsymbol{e}^{\mu} \boldsymbol{e}^{\mu}+\boldsymbol{e}^{\hat{\mu}} \boldsymbol{e}^{\hat{\mu}}\right) .
\end{aligned}
$$

The matrix power $\boldsymbol{Q}^{\cdot l}$ of the tensor $\boldsymbol{Q}$ is defined in Eq. (16). The Killing tensors are completely symmetric tensors obeying the equations

$$
\nabla_{(a} K_{b c)}^{(j)}=0
$$

Let us remark that the constant $c_{n}$ present in an odd number of spacetime dimensions is the square of the constant corresponding to the Killing vector $\boldsymbol{f}^{(n)}$; cf. Sections IV and V

The relation (19) can be inverted using the identities (A6) and (A7) from the Appendix. We obtain

$$
u_{\mu}^{2}+u_{\hat{\mu}}^{2}=U_{\mu}^{-1} \sum_{j=0}^{m}\left(-x_{\mu}^{2}\right)^{n-1-j} c_{j}
$$

and, in an odd number of dimensions,

$$
u_{\hat{0}}^{2}=\frac{c_{n}}{A^{(n)}} .
$$

Here, the quantity $U_{\mu}$ is defined as (cf. [18])

$$
U_{\mu} \equiv \prod_{\nu \neq \mu}\left(x_{\nu}^{2}-x_{\mu}^{2}\right)
$$

The coefficients $A^{(j)}$ of the $\beta$-expansion of $W_{0}(\beta)$ are the sums of all different products of $j$ different eigenvalues of $\boldsymbol{h}$, cf. Eq. (13). Such combinations can be obtained taking first the $j$-th wedge-power of the 2 -form $\boldsymbol{h}$,

$$
\boldsymbol{h}^{\wedge j}=\boldsymbol{h} \wedge \cdots \wedge \boldsymbol{h}=j ! \sum_{\nu_{1}<\cdots<\nu_{j}} x_{\nu_{1}} \ldots x_{\nu_{j}} \boldsymbol{\omega}^{\nu_{1}} \wedge \cdots \wedge \boldsymbol{\omega}^{\nu_{j}}
$$

and contracting it with itself in all tensor indices. Indeed,

$$
\begin{aligned}
A^{(j)} & =\frac{1}{(2 j) !(j !)^{2}} \boldsymbol{h}^{\wedge j} \bullet \boldsymbol{h}^{\wedge j} \\
& =\frac{(2 j) !}{\left(2^{j} j !\right)^{2}} h^{\left[a_{1} b_{1}\right.} \ldots h^{\left.a_{j} b_{j}\right]} h_{\left[a_{1} b_{1}\right.} \ldots h_{\left.a_{j} b_{j}\right]},
\end{aligned}
$$

where $\bullet$ denotes the complete contraction, i.e., $\boldsymbol{B} \bullet \boldsymbol{B}=$ $B_{a b c \ldots} B^{a b c \ldots}$, and where we used the orthogonality $\boldsymbol{\omega}^{\mu} \bullet \boldsymbol{\omega}^{\nu}=2 \delta^{\mu \nu}$, along with the normalization (A4).

Observing that the relation between the constants $w^{-1} c_{j}$ and the tensor $w^{-1} \boldsymbol{F}$ is the same as between $A^{(j)}$ and $\boldsymbol{h}$ [cf. Eqs. (4), (6), (7), (8), and (12)], we obtain a new simple expression for the constants of motion $c_{j}$,

$$
c_{j}=\frac{1}{(2 j) !(j !)^{2}} w^{1-2 j} \boldsymbol{F}^{\wedge j} \bullet \boldsymbol{F}^{\wedge j} .
$$

If one defines a 1-form $v$ with components

$$
v_{a}=h_{a b} u^{b}
$$

orthogonal to the velocity 1-form $\boldsymbol{u}$, then Eq. (2) implies that

$$
\boldsymbol{F}=w \boldsymbol{h}+\boldsymbol{u} \wedge \boldsymbol{v}
$$

Since $\boldsymbol{u} \wedge \boldsymbol{v} \wedge \boldsymbol{u} \wedge \boldsymbol{v}=0$, we have

$$
\boldsymbol{F}^{\wedge j}=w^{j} \boldsymbol{h}^{\wedge j}+j w^{j-1} \boldsymbol{u} \wedge \boldsymbol{v} \wedge \boldsymbol{h}^{\wedge(j-1)} .
$$

The total contraction of this with itself is

$$
\begin{aligned}
& \boldsymbol{F}^{\wedge j} \bullet \boldsymbol{F}^{\wedge j}=w^{2 j} \boldsymbol{h}^{\wedge j} \bullet \boldsymbol{h}^{\wedge j} \\
& \quad+2 j w^{2 j-1} \boldsymbol{h}^{\wedge j} \bullet\left(\boldsymbol{u} \wedge \boldsymbol{v} \wedge \boldsymbol{h}^{\wedge(j-1)}\right) \\
& \quad+j^{2} w^{2 j-2}\left(\boldsymbol{u} \wedge \boldsymbol{v} \wedge \boldsymbol{h}^{\wedge(j-1)}\right) \bullet\left(\boldsymbol{u} \wedge \boldsymbol{v} \wedge \boldsymbol{h}^{\wedge(j-1)}\right) .
\end{aligned}
$$

In the total contraction of the last term, $\boldsymbol{u} \cdot \boldsymbol{v}=0$, and any term with a contraction of $\boldsymbol{u}$ with any of the $\boldsymbol{h}$ 's gives another $\boldsymbol{v}$ which combines with the original $\boldsymbol{v}$ to give zero by the antisymmetry of the wedge product. Therefore, the only nonzero parts of the last term have another $w=\boldsymbol{u} \cdot \boldsymbol{u}$ factor, giving a total factor of $w^{2 j-1}$ for the total contraction of that term. Upon the substitution of Eq. (32) into (28), the dependence on $w$ cancels out, and we recover the quadratic dependence on $\boldsymbol{u}$ which enters through $\boldsymbol{u}$ and $\boldsymbol{v}$. Comparing with (20), we can write the tensorial relation between $\boldsymbol{K}^{(j)}$ and $\boldsymbol{h}$, which 
in components reads

$$
\begin{aligned}
K_{b}^{(j) a}= & \frac{(2 j) !}{\left(2^{j} j !\right)^{2}}\left(\delta_{b}^{a} h^{\left[a_{1} b_{1}\right.} \ldots h^{\left.a_{j} b_{j}\right]} h_{\left[a_{1} b_{1}\right.} \ldots h_{\left.a_{j} b_{j}\right]}\right. \\
& -4 j h^{\left[a b_{1}\right.} \ldots h^{\left.a_{j} b_{j}\right]} h_{b\left[b_{1}\right.} \ldots h_{\left.a_{j} b_{j}\right]} \\
& \left.+2 j h^{a\left[b_{1}\right.} \ldots h^{\left.a_{j} b_{j}\right]} h_{b\left[b_{1}\right.} \ldots h_{\left.a_{j} b_{j}\right]}\right) \\
= & \frac{(2 j) !}{\left(2^{j} j !\right)^{2}}\left(\delta_{b}^{a} h^{\left[a_{1} b_{1}\right.} \ldots h^{\left.a_{j} b_{j}\right]} h_{\left[a_{1} b_{1}\right.} \ldots h_{\left.a_{j} b_{j}\right]}\right. \\
& \left.-2 j h^{\left[a b_{1}\right.} \ldots h^{\left.a_{j} b_{j}\right]} h_{\left[b b_{1}\right.} \ldots h_{\left.a_{j} b_{j}\right]}\right)
\end{aligned}
$$

where we have employed the definition (29), the identities (A2) and (A3), and the normalization (A4).

Recently [21] there have been found different conserved quantities,

$$
C_{j}=w^{-j} \operatorname{tr}\left[\left(-F^{2}\right)^{j}\right],
$$

which are, however, not quadratic in velocities. Now we show that also these observables can be generated from the generating function $W(\beta)$. Taking the logarithm of Eq. (5) and expanding it into a power series, we obtain

$$
\log W(\beta)=\frac{1}{2} \operatorname{tr} \log \left(I-\beta w^{-2} F^{2}\right)=\sum_{j=0}^{\infty} \frac{(-1)^{j+1}}{2 j} \frac{\beta^{j}}{w^{j}} C_{j} .
$$

The constants $C_{j}$ are thus (up to constant factors and powers of $w$ ) given by derivatives of $\log W(\beta)$. We also obtained the relation between both sets of constants which can be formulated as

$$
\sum_{j=0}^{\infty} w^{-1} c_{j} \beta^{j}=\exp \left(\sum_{j=0}^{\infty} \frac{(-1)^{j+1}}{2 j} \frac{\beta^{j}}{w^{j}} C_{j}\right) .
$$

Comparing different orders of $\beta$ we get for the first four constants

$$
\begin{aligned}
c_{1} & =-\frac{1}{2} C_{1}, \\
w c_{2} & =-\frac{1}{4} C_{2}+\frac{1}{8} C_{1}^{2}, \\
w^{2} c_{3} & =-\frac{1}{6} C_{3}+\frac{1}{8} C_{1} C_{2}-\frac{1}{48} C_{1}^{3}, \\
w^{3} c_{4} & =-\frac{1}{8} C_{3}+\frac{1}{12} C_{1} C_{3}+\frac{1}{32} C_{2}^{2}-\frac{1}{32} C_{1}^{2} C_{2}+\frac{1}{384} C_{1}^{3} .
\end{aligned}
$$

It is shown in 22 that the observables $C_{j}$ Poisson commute between each other. The relation (36), which shows that the $c_{j}$ 's are polynomial combinations of the $C_{j}$ 's and $w$ with constant coefficients, thus proves that also the observables $c_{j}$ are in involution,

$$
\left\{c_{i}, c_{j}\right\}=0 .
$$

This gives non-trivial relations for the corresponding Killing tensors, namely

$$
K_{e(a}^{(j)} \nabla^{e} K_{b c)}^{(l)}-K_{e(a}^{(l)} \nabla^{e} K_{b c)}^{(j)}=0 .
$$

\section{KILLING-YANO TENSORS}

The existence of the closed conformal Killing-Yano tensor $\boldsymbol{h}$ guarantees the existence of the KillingYano tensor $\boldsymbol{f}$ which is obtained by the Hodge dual $\boldsymbol{f}=* \boldsymbol{h}$ [26]. This principal Killing-Yano tensor enables one to construct a rank-2 Killing tensor $K_{b}^{a}=f^{a e_{1} \ldots e_{D-3}} f_{b e_{1} \ldots e_{D-3}}$. Here we demonstrate that all the Killing tensors found in the previous section can be constructed in a similar way.

First, let us recall that a conformal Killing-Yano tensor (CKYT) [27, 28, 29] of a general rank $r$ is an antisymmetric $r$-form $\boldsymbol{f}$ the covariant derivative of which can be split into the antisymmetric and divergence parts

$$
\nabla \boldsymbol{f}=\mathcal{A} \nabla \boldsymbol{f}+\mathcal{T} \nabla \boldsymbol{f} .
$$

Here $\mathcal{A}$ is the standard anti-symmetrization and $\mathcal{T}$ is the projection onto the 'trace' part of the tensor of rank $r+1$ which is antisymmetric in the last $r$ indices,

$$
\mathcal{T} A_{a a_{1} \ldots a_{r}}=\frac{r}{D-r+1} g_{a\left[a_{1}\right.} A_{\left.|e| a_{2} \ldots a_{r}\right]}^{e} .
$$

The operation $\mathcal{T}$ satisfies $\mathcal{T}^{2}=\mathcal{T}$ and $\mathcal{T} \mathcal{A}=\mathcal{A} \mathcal{T}=0$. This means that a tensor $\boldsymbol{A}$ satisfies $\mathcal{T} \boldsymbol{A}=\boldsymbol{A}$ if and only if it has the form $A_{a a_{1} \ldots a_{r}}=g_{a\left[a_{1}\right.} \alpha_{\left.a_{2} \ldots a_{r}\right]}$. The divergence part $\mathcal{T} \nabla \boldsymbol{f}$ thus depends only on the divergence $\nabla_{e} f_{a b . . .}$. The condition (40) implies that $\nabla \boldsymbol{f}$ does not have a harmonic part [30] (given by the complement of the $\mathcal{A}$ and $\mathcal{T}$ projectors), i.e., $\boldsymbol{f}$ does not have a part for which both $\boldsymbol{d} \boldsymbol{f}$ and $\nabla \cdot \boldsymbol{f}$ vanishes.

A CKYT transforms into a CKYT under Hodge duality. The antisymmetric part $\mathcal{A} \nabla \boldsymbol{f}$ transforms into the divergence part $\mathcal{T} \nabla * \boldsymbol{f}$ and vice versa.

A Killing-Yano tensor $\boldsymbol{f}$ is such a CKYT for which the divergence part is missing, i.e.,

$$
\nabla \boldsymbol{f}=\mathcal{A} \nabla \boldsymbol{f} .
$$

The dual of a Killing-Yano tensor is a closed CKYT (see also [26]), i.e., an $r$-form obeying

$$
\nabla \boldsymbol{f}=\mathcal{T} \nabla \boldsymbol{f} .
$$

The wedge product of two closed CKYTs is again a closed CKYT [see Eq. (A5) in the Appendix]. We can thus start with the principal closed CKYT $\boldsymbol{h}$ and construct its wedge powers $\boldsymbol{h}^{\wedge j}(j=0, \ldots, k-1)$, which are again closed CKYTs. Their duals,

$$
\boldsymbol{f}^{(j)}=* \boldsymbol{h}^{\wedge j},
$$

are then Killing-Yano tensors of rank $D-2 j$. Their components are

$$
f_{a_{1} \ldots a_{D-2 j}}^{(j)}=2^{-j} \varepsilon_{a_{1} \ldots a_{D-2 j}} e_{1 \ldots e_{2 j}} h_{e_{1} e_{2}} \ldots h_{e_{2 j-1} e_{2 j}},
$$

where $\varepsilon_{a_{1} \ldots a_{D}}$ are components of the Levi-Civita tensor $\varepsilon$ and the normalization (A4) has been employed. 
Now we show that these Killing-Yano tensors generate the rank-2 Killing tensors $\boldsymbol{K}^{(j)}$ constructed above. Namely, using the identity (A1), we write

$$
\begin{aligned}
& \frac{1}{(D-2 j-1) !(j !)^{2}} f^{(j) a e_{1} \ldots e_{D-2 j}} f_{b e_{1} \ldots e_{D-2 j}}^{(j)}= \\
& =\frac{(2 j+1) !}{\left(2^{j} j !\right)^{2}} \delta\left[{ }_{b}^{a} h^{a_{1} b_{1}} \ldots h^{\left.a_{j} b_{j}\right]} h_{a_{1} b_{2}} \ldots h_{\left.a_{j} b_{j}\right]} .\right.
\end{aligned}
$$

With the help of Eq. (A2), we see that the last expression coincides with the formula (33) for the Killing tensors. So we have

$$
K_{b}^{(j) a}=\frac{1}{(D-2 j-1) !(j !)^{2}} f^{(j) a e_{1} \ldots e_{D-2 j}} f_{b e_{1} \ldots e_{D-2 j}}^{(j)} .
$$

Starting from the principal closed CKYT $\boldsymbol{h}$, we build the sequence of closed CKYTs $\boldsymbol{h}^{\wedge j}$, given explicitly in Eq. (26), which generates Killing-Yano tensors $\boldsymbol{f}^{(j)}=* \boldsymbol{h}^{\wedge j}$. These Killing-Yano tensors can be used to construct the rank-2 Killing tensors $\boldsymbol{K}^{(j)}$ given by the formula (47), or explicitly by Eq. (21) or (33).

In particular, in an odd number of spacetime dimensions, the last Killing-Yano tensor $\boldsymbol{f}^{(n)} \propto \sqrt{A^{(n)}} \boldsymbol{e}_{\hat{0}}$ is a Killing vector. Obviously, the corresponding Killing tensor $\boldsymbol{K}^{(n)} \propto \boldsymbol{f}^{(n)} \boldsymbol{f}^{(n)}$ is reducible.

\section{KERR-NUT-ADS SPACETIMES}

We shall now demonstrate that the structure explored above is fully realized in the Kerr-NUT-AdS spacetimes [18].

In the notation of previous sections (i.e., using the base of 1 -forms $\boldsymbol{e}^{a}=\left\{\boldsymbol{e}^{\mu}, \boldsymbol{e}^{\hat{\mu}}, \boldsymbol{e}^{\hat{0}}\right\}, \quad \mu=1, \ldots, n, \hat{\mu}=\mu+n$, $\hat{0}=2 n+1$, with the 1 -form $\boldsymbol{e}^{\hat{0}}$ present only for odd $D$ ), the Kerr-NUT-AdS metric may be written in the orthonormal form

$$
\boldsymbol{g}=\sum_{a=1}^{D} \boldsymbol{e}^{a} \boldsymbol{e}^{a}=\sum_{\mu=1}^{n}\left(\boldsymbol{e}^{\mu} \boldsymbol{e}^{\mu}+\boldsymbol{e}^{\hat{\mu}} \boldsymbol{e}^{\hat{\mu}}\right)+\varepsilon \boldsymbol{e}^{\hat{0}} \boldsymbol{e}^{\hat{0}}
$$

where the orthonormal basis one-forms are

$$
\begin{aligned}
\boldsymbol{e}^{\mu} & =Q_{\mu}^{-1 / 2} \boldsymbol{d} x_{\mu} \\
\boldsymbol{e}^{\hat{\mu}} & =Q_{\mu}^{1 / 2} \sum_{j=0}^{n-1} A_{\mu}^{(j)} \boldsymbol{d} \psi_{j} \\
\boldsymbol{e}^{\hat{0}} & =\left(-c / A^{(n)}\right)^{1 / 2} \sum_{j=0}^{n} A^{(j)} \boldsymbol{d} \psi_{j} .
\end{aligned}
$$

The quantities $A^{(j)}, A_{\mu}^{(j)}$ in terms of coordinates $x_{\mu}$ are of the form of Eqs. (13) and (18), $Q_{\mu}=X_{\mu} / U_{\mu}$ with $U_{\mu}$ given by Eq. (25), $c=\prod_{j=1}^{k-1} a_{j}^{2}$, and

$$
X_{\mu}=(-1)^{1-\varepsilon} \frac{1+\lambda x_{\mu}^{2}}{x_{\mu}^{2 \varepsilon}} \prod_{j=1}^{k-1}\left(a_{j}^{2}-x_{\mu}^{2}\right)+2 M_{\mu}\left(-x_{\mu}\right)^{1-\varepsilon}
$$

The constants $\left(M_{\mu}, a_{j}\right)$ are related to the mass, NUT parameters, and angular momenta, and $\lambda$ is proportional to the cosmological constant [18]. The metric represents an Einstein space obeying the Einstein equation

$$
R_{a b}=(D-1) \lambda g_{a b}
$$

Using the identities (A6) and (A7), we find the dual vectors

$$
\begin{aligned}
& \boldsymbol{e}_{\mu}=Q_{\mu}^{1 / 2} \boldsymbol{\partial}_{x_{\mu}} \\
& \boldsymbol{e}_{\hat{\mu}}=\frac{1}{Q_{\mu}^{1 / 2} U_{\mu}} \sum_{j=0}^{k-1}\left(-x_{\mu}^{2}\right)^{n-1-j} \boldsymbol{\partial}_{\psi_{j}} \\
& \boldsymbol{e}_{\hat{0}}=\left(-c A^{(n)}\right)^{-1 / 2} \boldsymbol{\partial}_{\psi_{n}}
\end{aligned}
$$

and the corresponding inverse relations

$$
\begin{aligned}
\boldsymbol{\partial}_{x_{\mu}} & =Q_{\mu}^{-1 / 2} \boldsymbol{e}_{\mu} \\
\boldsymbol{\partial}_{\psi_{j}} & =\varepsilon A^{(j)}\left(-\frac{c}{A^{(n)}}\right)^{1 / 2} \boldsymbol{e}_{\hat{0}}+\sum_{\mu=1}^{n} Q_{\mu}^{1 / 2} A_{\mu}^{(j)} \boldsymbol{e}_{\hat{\mu}}, \\
\boldsymbol{\partial}_{\psi_{n}} & =\left(-c A^{(n)}\right)^{1 / 2} \boldsymbol{e}_{\hat{0}} .
\end{aligned}
$$

It is possible to prove [31] that that the $\boldsymbol{h}$ found in [20] (there called $\boldsymbol{k}$ ) in this metric in all dimensions $D$ is a principal closed CKYT, which in the frame (49) takes exactly the form (10). This means that the generally defined eigenvalues $x_{\mu}$ of the principal CKYT $\boldsymbol{h}$ (10) coincide with the chosen ('natural') coordinates $x_{\mu}$ of the Kerr-NUT-AdS metric.

We now demonstrate that from the very existence of this tensor one can extract all the constants of geodesic motion for the Kerr-NUT-AdS metrics. Namely, besides the $k$ constants of motion (19) connected with the rank-2 Killing tensors (21), also all the $k$ isometries follow from the existence of the principal CKYT $\boldsymbol{h}$.

First of all, it was proved in [32] that in an Einstein space, obeying Eq. (51), the divergence $\boldsymbol{\xi}$ of a CKYT $\boldsymbol{h}$,

$$
\xi^{a}=\frac{1}{D-1} \nabla_{b} h^{b a},
$$

is a Killing vector. In particular we find $\boldsymbol{\xi}=\boldsymbol{\partial}_{\psi_{0}}$ [20].

Next, using Eqs. (21) and (53), we can recover $n-1$ other Killing vectors $\boldsymbol{\partial}_{\psi_{j}}$,

$$
\left(\partial_{\psi_{j}}\right)^{a}=K_{b}^{(j) a} \xi^{b}, \quad j=1, \ldots, n-1 .
$$

For a similar construction in 4D see [33, 34, 35]. Finally, as mentioned in Section IV] in odd dimensions the last Killing vector is given by the $n$-th Killing-Yano tensor $\boldsymbol{f}^{(n)}$, which in the present example turns out to be $\boldsymbol{\partial}_{\psi_{n}}$.

It would be very interesting to find under what general conditions on the CKYT $\boldsymbol{h}$, and possibly on the curvature, this construction gives all the isometries present in the spacetime. 
The constants of geodesic motion in the higherdimensional Kerr-NUT-AdS spacetime are now completely determined. Denoting the constants from the Killing vectors as

$$
b_{j}=\left(\partial_{\psi_{j}}\right)^{a} u_{a}, \quad j=0, \ldots, k-1,
$$

we first find the frame components of velocity $u_{\hat{\mu}}$, and possibly $u_{\hat{0}}$. It follows from Eq. (52) that

$u_{\hat{\mu}}=\frac{1}{Q_{\mu}^{1 / 2} U_{\mu}} \sum_{j=0}^{k-1}\left(-x_{\mu}^{2}\right)^{n-1-j} b_{j}, u_{\hat{0}}=\left(-c A^{(n)}\right)^{-1 / 2} b_{n}$.

Comparing with (24) we find

$$
c_{n}=-\frac{b_{n}^{2}}{c},
$$

which clearly illustrates the fact that the constant $c_{n}$ corresponds to the reducible Killing tensor $\boldsymbol{K}^{(n)} \propto \boldsymbol{f}^{(n)} \boldsymbol{f}^{(n)}$. The remaining components of velocity, $u_{\mu}$, are given (up to signs) in terms of the constants $b_{j}, j=0, \ldots, k-1$, and $c_{j}, j=0, \ldots, n-1$ [which correspond to the irreducible Killing tensors (21)] by Eq. (23).

The existence of $n$ rank-2 irreducible Killing tensors $\boldsymbol{K}^{(j)}$ and $k=D-n$ Killing vectors $\boldsymbol{\partial}_{\psi_{j}}$ is closely related to the question of separability of the Hamilton-Jacobi and Klein-Gordon equations. It is shown in [36, 37] that if the spacetime possesses such tensors that satisfy the condition (39), $\mathcal{L}_{\boldsymbol{\partial}_{\psi_{j}}} \boldsymbol{K}^{(j)}=0$ with $\left\{\boldsymbol{\partial}_{\psi_{j}}, \boldsymbol{\partial}_{\psi_{l}}\right\}=0$ which holds in our case - then there exists a so-called separability structure. This structure guarantees the separability of the Hamilton-Jacobi equation and for Einstein spaces also the separability of the Klein-Gordon equation.

The separability of these equations was explicitly demonstrated recently 23]. It turns out that the integration constants obtained by the separation of the Hamilton-Jacobi equation are the quantities $c_{j}$ given by Eq. (19). Indeed, if we transform the tetrad components $u_{\mu}, u_{\hat{\mu}}$ and $u_{\hat{0}}$ into the coordinate frame we find that the expression $U_{\mu}\left(u_{\mu}^{2}+u_{\hat{\mu}}^{2}\right)$ corresponds to the quantity $F_{\mu}$ of 23]. Comparing Eqs. (23) and (14) of 23], we can identify the constants defined above with those from 23] (cf. also Eqs. (24) and (58) with Eq. (15) of [23]).

\section{DISCUSSION}

We have seen that the existence of a principal KillingYano tensor, one of rank $D-2$, guarantees the existence of $k=D-n$ Killing-Yano tensors of $\operatorname{rank}(D-2 j)$, $j=0, \ldots, k-1$, and that each of these Killing-Yano tensors generates a Killing tensor of rank 2, $n$ of which are irreducible. In the case of the Kerr-NUT-AdS spacetimes, the principal Killing-Yano tensor also generates all the $k$ Killing vectors, and hence all $D$ of the independent constants of geodesic motion.
Our results raise various questions. For example, is the construction of Killing vectors by a rank- $(D-2)$ KillingYano tensor general, or specific to only certain metrics? For what classes of spacetimes are there rank- $(D-2)$ Killing-Yano tensors? For what classes of such spacetimes does the rank- $(D-2)$ Killing-Yano tensor generate enough Killing tensors to give $D$ independent constants of motion? Are there any new Einstein metrics within these classes? Do these structures enable one to separate the Dirac, electromagnetic, and gravitational wave equations in the Kerr-NUT-AdS spacetimes and/or in any other possible members of these classes? What is the relation of the existence of principal Killing-Yano tensors to the algebraic type of the metric? We suspect that our observations may be the tip of an iceberg of important new relations for higher dimensional spacetime metrics.

\section{Acknowledgments}

P.K. is supported by the grant GAČR 202/06/0041 and appreciates the hospitality of the University of Alberta. D.K. is grateful to the Golden Bell Jar Graduate Scholarship in Physics at the University of Alberta. D.P. thanks the Natural Sciences and Engineering Research Council of Canada for financial support. V.F. thanks the Natural Sciences and Engineering Research Council of Canada and the Killam Trust for financial support. We have benefited from discussions with Paul Davis and Gary Gibbons.

\section{APPENDIX A: USEFUL IDENTITIES}

In this Appendix we list some identities used in the main text. Their proofs are mostly straightforward but also lengthy and cumbersome.

First we list three identities for antisymmetric tensors. The Levi-Civita tensor satisfies

$$
\varepsilon^{a_{1} \ldots a_{r} c_{r+1} \ldots c_{D}} \varepsilon_{b_{1} \ldots b_{r} c_{r+1} \ldots c_{D}}=r !(D-r) ! \delta_{b_{1}}^{\left[a_{1}\right.} \ldots \delta_{b_{r}}^{\left.a_{r}\right]},
$$

the projector on the antisymmetric tensors can be split as

$$
(r+1) \delta_{[b}^{[a} \delta_{b_{1}}^{a_{1}} \ldots \delta_{\left.b_{r}\right]}^{\left.a_{r}\right]}=\delta_{b}^{a} \delta_{\left[b_{1}\right.}^{\left[a_{1}\right.} \ldots \delta_{\left.b_{r}\right]}^{\left.a_{r}\right]}-r \delta_{\left[b_{1}\right.}^{a} \delta_{|b|}^{\left[a_{1}\right.} \ldots \delta_{\left.b_{r}\right]}^{\left.a_{r}\right]}
$$

and finally

$$
h_{\left[a_{1} b_{1}\right.} \ldots h_{\left.a_{j} b_{j}\right]}=h_{a_{1}\left[b_{1}\right.} \ldots h_{\left.a_{j} b_{j}\right]},
$$

which holds for any antisymmetric tensor $h_{a b}$.

In our convention the wedge product is proportional to the anti-symmetrization of the tensor product which for the $j$-th wedge-power of a 2 -form $\boldsymbol{h}$ gives

$$
\boldsymbol{h}^{\wedge j}=\frac{(2 j) !}{(2 !)^{j}} \mathcal{A} \boldsymbol{h}^{j} .
$$


Next we want to show that the wedge product of two closed CKYTs $\boldsymbol{p}$ and $\boldsymbol{q}$ of rank $r$ and $s$ respectively is again a closed CKYT. It is clear that $\boldsymbol{p} \wedge \boldsymbol{q}$ is closed. Rewriting the wedge product $\boldsymbol{p} \wedge \boldsymbol{q}$ as the antisymmetrization of their tensor product, we get

$$
\begin{aligned}
\nabla_{e}\left(p_{[a b \ldots} q_{c d \ldots]}\right)= & \left(\nabla_{e} p_{[a b \ldots}\right) q_{c d \ldots]}+p_{[a b \ldots}\left(\nabla_{|e|} q_{c d \ldots]}\right) \\
=g_{e[a}\left(\frac{r}{D-r+1}(\right. & \left.\nabla_{|g|} p^{g} b \ldots\right) q_{c d \ldots]} \\
& \left.\left.+(-1)^{r} \frac{s}{D-s+1} p_{b \ldots c}\left(\nabla_{|g|} q^{g} d \ldots\right]\right)\right)
\end{aligned}
$$

where we used the property (43) of $\boldsymbol{p}$ and $\boldsymbol{q}$. We can see by inspection that the result has the form $g_{a\left[a_{1}\right.} \alpha_{\left.a_{2} \ldots a_{r}\right]}$, so, as we discussed after Eq. (41), $\mathcal{T}(\boldsymbol{p} \wedge \boldsymbol{q})=\boldsymbol{p} \wedge \boldsymbol{q}$, and hence $\boldsymbol{p} \wedge \boldsymbol{q}$ is a closed CKYT.
If we understand $A_{\mu}^{(j)}$ with $\mu=1, \ldots, n$ and $j=0, \ldots, n-1$ as an $n \times n$ matrix, its inverse is $B_{(j)}^{\mu}=\left(-x_{\mu}^{2}\right)^{n-1-j} / U_{\mu}$ with $U_{\mu}$ defined in Eq. (50). This means

$$
\sum_{j=0}^{n-1}\left(-x_{\mu}^{2}\right)^{n-1-j} \frac{A_{\nu}^{(j)}}{U_{\mu}}=\delta_{\mu}^{\nu}, \quad \sum_{\mu=1}^{n}\left(-x_{\mu}^{2}\right)^{n-1-j} \frac{A_{\mu}^{(l)}}{U_{\mu}}=\delta_{j}^{l} .
$$

We also have its 'extension' for $j=n, l=0, \ldots, n-1$ :

$$
\sum_{\mu=1}^{n} \frac{A_{\mu}^{(l)}}{x_{\mu}^{2} U_{\mu}}=\frac{A^{(l)}}{A^{(n)}} .
$$

[1] R. P. Kerr, Phys. Rev. Lett. 11, 237 (1963).

[2] B. Carter, Commun. Math. Phys. 10, 280 (1968).

[3] P. Stackel, C. R. Acad. Sci. Paris 121, 489 (1895).

[4] M. Walker and R. Penrose, Commun. Math. Phys. 18, 265 (1970).

[5] K. Yano, Ann. Math. 55, 328 (1952).

[6] R. Penrose, Ann. N. Y. Acad. Sci. 224, 125 (1973);

[7] R. Floyd, "The Dynamics of Kerr Fields," Ph.D. Thesis, London (1973).

[8] S. A. Teukolsky, Astrophys. J. 185, 635 (1973).

[9] W. Unruh, Phys. Rev. Lett. 31, 1265 (1973).

[10] S. Chandrasekhar, Proc. Roy. Soc. London A349, 571 (1976).

[11] D. N. Page, Phys. Rev. D 14, 1509 (1976).

[12] S. A. Teukolsky, Phys. Rev. Lett. 29, 1114 (1972).

[13] R. C. Myers and M. J. Perry, Ann. Phys. 172, 304 (1986).

[14] S. W. Hawking, C. J. Hunter, and M. M. TaylorRobinson, Phys. Rev. D 59064005 (1999), hep-th/9811056

[15] G. W. Gibbons, H. Lü, D. N. Page and C. N. Pope, J. Geom. Phys. 53, 49 (2005), hep-th/0404008

[16] G. W. Gibbons, H. Lü, D. N. Page and C. N. Pope, Phys. Rev. Lett. 93, 171102 (2004), hep-th/0409155.

[17] Z. W. Chong, G. W. Gibbons, H. Lü, and C. N. Pope, Phys. Lett. B 609, 124 (2005), hep-th/0405061.

[18] W. Chen, H. Lü and C. N. Pope, Class. Quant. Grav. 23, 5323 (2006), hep-th/0604125

[19] V. P. Frolov and D. Kubizňák, "Hidden Symmetry of Higher Dimensional Rotating Black Holes," Phys. Rev. Lett. (to appear), gr-qc/0605058

[20] D. Kubizñák and V. P. Frolov, "Hidden Symmetry of Higher Dimensional Kerr-NUT-AdS Spacetimes," gr-qc/0610144.

[21] D. N. Page, D. Kubizňák, M. Vasudevan, and P. Krtouš, "Complete Integrability of Geodesic Motion in General Kerr-NUT-AdS Spacetimes," hep-th/0611083

[22] P. Krtouš, D. Kubizńák, D. N. Page, and M. Vasudevan, (in preparation).

[23] V. P. Frolov, P. Krtouš, and D. Kubizňák, "Separability of Hamilton-Jacobi and Klein-Gordon Equations in General Kerr-NUT-AdS Spacetimes," hep-th/0611245.

[24] In the construction presented here we do not assume the specific form of the metric; however, similar to the KerrNUT-AdS case [18], we assume that the metric is written at least formally with Euclidean signature. The Wick ro- tation to physical signature would bring some different signs in various expressions, but the general structure would not be affected.

[25] The velocity $\boldsymbol{u}$ appears here in a dual way. When proving that, e.g., $\boldsymbol{F}$ is covariantly conserved along each geodesic, we understand $\boldsymbol{u}$ as the tangent vector with respect to an affine parameter (not necessary normalized). However, we can also use the velocity $\boldsymbol{u}$ in a situation when we do not assume the whole trajectory to which $\boldsymbol{u}$ is tangent. In such a case we refer to a point in the phase space which is naturally realized as the cotangent bundle. The point in the phase space is given by the point in the configuration space and by the momentum 1-form which can be identified with the unnormalized velocity. Indeed, the geodetic motion is generated by the Hamiltonian $w=\boldsymbol{u} \cdot \boldsymbol{u}$, the momentum is $\boldsymbol{u}$, and conserved quantities are those which Poisson commute with $w$.

[26] M. Cariglia, Class. Quant. Grav. 21, 1051 (2004).

[27] S. Tachibana, Tôhoku Math. J. 21, 56 (1969).

[28] T. Kashiwada, Nat. Sci. Rep., Ochanomizu University 19, 67 (1968).

[29] S. Tachibana and T. Kashiwada. J. Math. Soc. Japan 21, 259 (1969).

[30] K. Yano and S. Bochner, Curvature and Betti numbers, (Princeton University Press, Princeton, 1953).

[31] This was checked in [20] for $D<10$ and proved by the authors for general dimensions $D$ by checking the condition (42) for the dual $\boldsymbol{f}=* \boldsymbol{h}$, using a direct calculation of the connection coefficients. Cf. also [38] for a recent independent calculation of the connection coefficients.

[32] J. Jezierski, Class. Quant. Grav. 14, 1679 (1997).

[33] L. P. Hughston and P. Sommers, Comm. Math. Phys. 33, 129 (1973).

[34] C. D. Collinson and P. N. Smith, Comm. Math. Phys. 56, 277 (1977).

[35] W. Dietz and R. Rüdiger, Proc. Roy. Soc. Lond. A375, 361 (1981).

[36] S. Benenti and M. Francaviglia, Gen. Rel. Grav. 10, 79 (1979).

[37] M. Demianski and M. Francaviglia, J. Theor. Phys. 19, 675 (1980).

[38] N. Hamamoto, T. Houri, T. Oota, and Y. Yasui, "Kerr-NUT-de Sitter Curvature in All Dimensions," hep-th/0611285. 